\title{
GLASMA EVOLUTION IN PARTONIC MEDIUM
}

\author{
A. V. Nazarenko \\ Bogolyubov Institute for Theoretical Physics, \\ 14-b, Metrologichna St., Kyiv,03680,Ukraine,nazarenko@bitp.kiev.ua
}

(Received October 5, 2010)

\begin{abstract}
We examine a scenario of the abelianized Glasma evolution with accounting for back-reaction of partonic medium in ultrarelativistic heavy-ion collisions. We announce that such a generalization leads to the instabilities and the presence of negative color conductivity in the system.
\end{abstract}

Key words: heavy-ion collisions, negative color conductivity.

PACS number(s): 24.85.+p, 41.20.Jb

\section{INTRODUCTION}

Phenomenological analyses of experimental data indicate that the quark-gluon plasma (QGP) can be formed in ultrarelativistic A+A collisions [1]. Its local thermalization and isotropization should be mainly related to the fast processes stimulated by instabilities at small times after collision $[2,3]$.

In the present theoretical picture of ultrarelativistic heavy-ion collisions [4], the early stage is preferably characterized by the large number of partons with "small" momenta of the order of the so-called saturation momentum $\Lambda_{s}$, which are better viewed as a classical YangMills field in vacuum [5], sometimes named as "Glasma". The initial conditions for Glasma evolution are determined by the Color Glass Condensate (CGC) concept by McLerran-Venugopalan (MV) [6], where the field sources before collision can be presented by the randomly distributed valent quarks of colliding hadrons and are located (due to Lorentz contraction) on infinitesimally thin sheets running along the light-cone. These sources are also treated as the hard partons with "large" momenta, which escape quickly from the system after collision. Thus, the original MV model neglects the interaction between the field and the hard partonic medium.

The space-time dynamics of the Yang-Mills fields in vacuum ("the melting of CGC") in assumption of boost invariance was investigated numerically, and the energy and the number distributions of the classically produced gluons were computed (see, for example, review [7]). Moreover, it was shown that the violations of boost invariance cause a non-Abelian Weibel instability [8] leading the field (soft) modes to grow with proper time [9]. However, the effect of isotropization is out of this model.

On the other hand, the hard partons (produced after moment of collision) with large transverse momentum $p_{T}$ can be studied within the framework of transport theory, and if the presence of the soft classical field is neglected, the time evolution of the partons is described by
Boltzmann equation with a collision kernel [10-15] (for comment, see ${ }^{1}$ ). However, it has been argued that the collective processes caused by the soft gauge field should be dominant in equilibration of QGP instabilities developed due to anisotropic distributions of released hard partons [16-19].

The third regime where the back-reaction of the field on the hard partons (treated as particles) is still weak but where the self-interaction of the former may be strongly nonlinear is governed by a "hard-loop" effective action which has been derived in Ref. [20] for arbitrary momentum-space anisotropies.

It is interesting to note that the numerical studies of anisotropic hard partonic modes coupled to unstable soft modes revealed the tendency of the non-Abelian gauge fields to "abelianize" during the stage of instabilities $[19,21]$. It means that the field commutators become much smaller than the fields themselves. Moreover, the dynamics of the Abelian and non-Abelian fields is qualitatively the same, if these fields are not strong enough.

In this paper, we examine a scenario of Glasma evolution with CGC-like initial conditions, when the presence of the (momentum-)anisotropic medium of hard partons is also taken into account. Our goal is to evaluate analytically the behavior of such a system in short-time interval in weak-interaction regime when the application of the abelianized version of the field dynamics is possible. Although the last condition demands to consider the system at relatively large times after $\mathrm{A}+\mathrm{A}$ collision (as follows from numerical investigations) but simplifies the problem considerably. However, it is already pointed out in Refs. [22-24] that the early equilibration of QGP is not necessary to describe pion and kaon spectra observed experimentally at RHIC in Brookhaven.

Since the momentum-space anisotropy of the system can be estimated by means of transport coefficients, we attempt to calculate a conductivity tensor and to determine an effect of instabilities on it. It is expected that the

\footnotetext{
${ }^{1}$ This list of references contains also some works on approaches based on the relaxation time approximation to the Boltzmann equation.
} 
back-reaction can lead to a negative color conductivity in the boost-invariant case.

\section{THE MODEL FORMULATION}

As was mentioned in the Introduction, the classical Yang-Mills theory in space-time with pseudo-cylindrical metric

$$
\begin{aligned}
& d s^{2}=d \tau^{2}-\tau^{2} d \eta^{2}-d r_{T}^{2}-r_{T}^{2} d \varphi^{2} \\
& \tau=\sqrt{t^{2}-z^{2}}, \quad \eta=\frac{1}{2} \ln \frac{t+z}{t-z}
\end{aligned}
$$

( $\tau$ and $\eta$ are proper time and space-time rapidity, respectively) has been abelianized since $\tau_{0} \approx 3 / \Lambda_{s}$, where $\Lambda_{s} \approx 2 \mathrm{GeV}$ [5]. It means that we actually come to the Maxwell theory with 4-potential $A_{\mu}$ (hereafter, we neglect the normalization constant $1 / \sqrt{N_{c}}$, where $N_{c}$ is the number of colors).

The free-field theory in mid-rapidity region in the case of central collisions, when the potentials are parametrized as $A_{\tau}=0$ (CGC-like gauge fixing), $A_{\eta} \equiv$ $\Phi\left(\tau, r_{T}\right), A_{r_{T}}=0, A_{\varphi} \equiv \Psi\left(\tau, r_{T}\right)$, has been already examined (see Ref. [23]) in order to describe the space-time evolution of the field flow (collective velocity) at prethermal stage of collisions. It turns out that the results obtained are qualitatively the same like in the case of non-Abelian model from Ref. [25].

Here we generalize the abelianized Glasma equations by the inclusion of sources in the right-hand side:

$$
\begin{aligned}
& \partial_{\tau}^{2} \Phi-\frac{1}{\tau} \partial_{\tau} \Phi-\partial_{r_{T}}^{2} \Phi-\frac{1}{r_{T}} \partial_{r_{T}} \Phi=J_{\eta}\left(\tau, r_{T}\right), \\
& \partial_{\tau}^{2} \Psi+\frac{1}{\tau} \partial_{\tau} \Psi-\partial_{r_{T}}^{2} \Psi+\frac{1}{r_{T}} \partial_{r_{T}} \Psi=J_{\varphi}\left(\tau, r_{T}\right) .
\end{aligned}
$$

Note that $J_{\tau}=0, J_{r_{T}}=0$ and the current conservation is satisfied automatically.

In the context of $\mathrm{A}+\mathrm{A}$ collisions, the presence of the sources corresponds to the existence of the medium. Accounting for a hard partonic component (viewed as a particle subsystem), we aim to investigate the field and particle dynamics.

The components of the current in Minkowskian spacetime are

$$
J^{\mu}=g \int p^{\mu}\left(f_{+}-f_{-}\right) \frac{d^{3} p}{p^{0}}
$$

where $p^{0} \equiv|\mathbf{p}|$ (the case of massless partons), $f_{ \pm}$are distribution functions of (scalar) partons. The distribution of partons is supposed to be anisotropic in the momentum space and inhomogeneous in configuration one. Space-time development of functions $f_{ \pm}$is determined by Vlasov equations which we will formulate below. Note that " $-g$ " corresponds to the charge of electron in the context of electrodynamics.

A toy field model with non-trivial right-hand side has been already investigated in Ref. [26].
It is useful to parametrize momenta as $\left(p^{\mu}\right)=$ $\left(p_{T} \cosh y, p_{T} \cos \phi, p_{T} \sin \phi, p_{T} \sinh y\right)$, where $y$ is momentum rapidity.

In terms of our variables one has:

$$
\begin{aligned}
& J_{\tau}=g \int p_{T}^{2} \cosh \theta\left(f_{+}-f_{-}\right) d p_{T} d y d \phi, \\
& J_{\eta}=-\tau g \int p_{T}^{2} \sinh \theta\left(f_{+}-f_{-}\right) d p_{T} d y d \phi, \\
& J_{r_{T}}=-g \int p_{T}^{2} \cos \xi\left(f_{+}-f_{-}\right) d p_{T} d y d \phi, \\
& J_{\varphi}=-r_{T} g \int p_{T}^{2} \sin \xi\left(f_{+}-f_{-}\right) d p_{T} d y d \phi,
\end{aligned}
$$

where $\theta=y-\eta, \xi=\phi-\varphi$.

Taking conditions $J_{\tau}=0, J_{r_{T}}=0$ into account the difference $f_{+}-f_{-}$should be an odd function of $\theta$ and $\xi$ during evolution.

The evolution of $f_{ \pm}$is generated by Vlasov equations:

$$
(\hat{L} \pm g \hat{F}) f_{ \pm}=0
$$

where $\hat{L} \equiv p^{\mu} \partial_{\mu}, \hat{F} \equiv p^{\mu} F_{\mu \nu} \partial_{p}^{\nu}, F_{\mu \nu}=\partial_{\mu} A_{\nu}-\partial_{\nu} A_{\mu}$.

Since the sources (partons) are randomly distributed at the initial moment $\tau_{0}$, we put $f_{+}^{0}=f_{-}^{0}=f^{0}$, where $f^{0}$ is defined as $\left.\left(d N_{h} / d^{3} x d^{3} p\right)\right|_{\tau_{0}}$, and $\hat{L} f^{0}=0$ in our investigations. It means that the system is neutral and the currents are absent at $\tau_{0}$.

Using the curved coordinates, we obtain

$$
\begin{aligned}
& \hat{L}=p_{T}\left(\cosh \theta \partial_{\tau}+\frac{\sinh \theta}{\tau} \partial_{\eta}+\cos \xi \partial_{r_{T}}+\frac{\sin \xi}{r_{T}} \partial_{\varphi}\right), \\
& \hat{F}=-\frac{\partial_{\tau} \Phi}{\tau} \partial_{y}+\frac{\partial_{r_{T}} \Phi}{\tau}\left(\sinh \theta \sin \xi \partial_{\phi}-\cosh \theta \cos \xi \partial_{y}\right) \\
& +\frac{\partial_{\tau} \Psi}{r_{T}}\left(\sinh \theta \sin \xi \partial_{y}+\cosh \theta \cos \xi \partial_{\phi}\right)+\frac{\partial_{r_{T}} \Psi}{r_{T}} \partial_{\phi} .
\end{aligned}
$$

We can see that the operator of Lorentz force $\hat{F}$ is simply the operator of rotation in momentum space and, therefore, conserves the absolute value of transverse momentum $p_{T}$. It is expected that such a structure of the Lorentz force should lead to the momentum transmission between different directions and, consequently, to instabilities in this system.

\section{SOLUTION OF EQUATIONS}

In this Section, we concentrate on finding the solution of the set of the coupled Maxwell-Vlasov equations. Since the Glasma field is essential at early stage of nuclear collisions (in contrast with the hard partons or quarks), the study of the field dynamics actually dominates. By this way, it is necessary to express the particle currents through the fields. In general, the Vlasov equations are complicated. For this reason, we are forced to use a method for approximate solution of this set.

A fluctuation of the distribution function, which arises during a fairly small time interval $\Delta \tau$ can be found in the linear approximation in $g$ :

$$
f_{ \pm}=f^{0} \mp g \delta f .
$$


In this approximation, the space-time evolution of correction $\delta f$, determining the difference $f_{+}-f_{-}=-2 g \delta f$ and the current components, results from the following equation:

$$
\hat{L} \delta f=\hat{F} f^{0}
$$

Note that this approximation does not permit us to investigate an isotropization of the particle (hard parton) kinematic part of the energy-momentum tensor, proportional to the sum $f_{+}+f_{-}$. It is expected that such isotropization effect can be observed if the correction of the order $g^{2}$ is included. Nevertheless, the approximation under consideration allows one to study the instabilities in the system.

If $\tau \rightarrow \tau_{0}$, there exists an approximate solution, which is short-living in time and localized in space,

$$
\delta f \approx \frac{\tau-\tau_{0}}{p_{T} \cosh \theta} \hat{F} f^{0} .
$$

It is easy to verify that the action of evolution operator $\hat{L}$ on this expression gives us

$$
\hat{L} \frac{\tau-\tau_{0}}{p_{T} \cosh \theta} \hat{F} f^{0}=\hat{F} f^{0}+\left(\tau-\tau_{0}\right) W(\tau),
$$

where

$$
\begin{aligned}
W(\tau) & =\left[\partial_{\tau}+\frac{\tanh \theta}{\tau}\left(\partial_{\eta}+\tanh \theta\right)\right. \\
& \left.+\frac{\cos \xi}{\cosh \theta} \partial_{r_{T}}+\frac{\sin \xi}{r_{T} \cosh \theta} \partial_{\varphi}\right]\left(\hat{F} f^{0}\right)
\end{aligned}
$$

Thus, if $\tau \rightarrow \tau_{0}$, the last term in right-hand side of (16) vanishes. Also note that this is the simplified proof of Eq. (15). To understand this approximation in details see Appendix A.

Often the model initial boost-invariant distributions in central heavy-ion collisions take the form $f^{0}=$ $f^{0}\left(p_{T}, \theta\right)=f^{0}\left(p_{T},-\theta\right)$ (note that for the sake of correctness $f^{0}$ has to be also a function of $\left.r_{T}\right)$. In this case, we obtain

$$
J_{\eta}=\sigma_{\eta}(\tau) \partial_{\tau} \Phi, \quad J_{\varphi}=\sigma_{\varphi}(\tau) \partial_{\tau} \Psi
$$

where

$$
\sigma_{\eta}(\tau)=2\left(\tau-\tau_{0}\right) \sigma_{0}, \quad \sigma_{\varphi}(\tau)=-\left(\tau-\tau_{0}\right) \sigma_{0}
$$

are conductivities.

The common multiplier dependent on the initial distribution of partons is

$$
\sigma_{0} \equiv-2 \pi g^{2} \int_{0}^{\infty} d p_{T} \int_{-\infty}^{\infty} d y \partial_{y} f^{0} p_{T} \tanh \theta
$$

We can immediately see that $\sigma_{\eta}(\tau)$ and $\sigma_{\varphi}(\tau)$ have the different signs. It says about the presence of negative color conductivity driving to instability in the system. The mechanism of this instability looks simple: we deal with situation when the particles (partons) give the energy to the field.

Now let us analyze the properties of $\sigma_{0}$. Firstly, we assume that the initial distribution $f^{0}$ is the product of the function of $\left(p_{T}, \theta\right)$ and the spatial distribution $\left.\left(d N_{h} / d^{3} x\right)\right|_{\tau_{0}}\left(r_{T}\right)$ in a transverse plane. Taking into account that the initial distribution is even function of $\theta$, one gets

$$
\sigma_{0}=\left.A \frac{d N_{h}}{d^{3} x}\right|_{\tau_{0}}>0,
$$

where $A$ is a positive constant arising after integration over momentum variables.

Thus, $\sigma_{\varphi}<0$, while $\sigma_{\eta}>0$. It means that the color negative conductivity takes place in the transverse plane.

At this stage the natural question arises: how does negative conductivity look in the laboratory reference frame. Eqs. (18) are actually the Ohm law, where $E_{\eta} \equiv F_{\tau \eta}=\partial_{\tau} \Phi, E_{\varphi} \equiv F_{\tau \varphi}=\partial_{\tau} \Psi$ are the (chromo)electric field strengths. Introducing $E_{i}=F_{t i}$ in the Minkowskian space-time, we find that $E_{\eta}=\tau E_{z}, E_{\varphi}=$ $r_{T} \cosh \eta\left(-\sin \varphi E_{x}+\cos \varphi E_{y}\right)+r_{T} \sinh \eta\left(-\sin \varphi F_{z x}+\right.$ $\left.\cos \varphi F_{z y}\right)$. In these terms the current components are

$$
\begin{aligned}
J_{t} & =-\sinh \eta \sigma_{\eta} E_{z}, \quad J_{z}=\cosh \eta \sigma_{\eta} E_{z}, \\
J_{x} & =-\frac{\sin \varphi}{r_{T}} \sigma_{\varphi} E_{\varphi}, \quad J_{y}=\frac{\cos \varphi}{r_{T}} \sigma_{\varphi} E_{\varphi} .
\end{aligned}
$$

If $\eta=0$ and $\varphi=0$, one has that $J_{t}=J_{x}=0$, $J_{y}=\sigma_{\varphi} E_{y}, J_{z}=\sigma_{\eta} E_{z}$. Thus the color negative conductivity takes place under some conditions (related with the value of angles) in the laboratory reference frame. This effect is associated with filamentation in the plasma [3].

Since it is hard to find the general solution of field equations for an arbitrary distribution $\left.\left(d N_{h} / d^{3} x\right)\right|_{\tau_{0}}$, we try to study the particular case, when $\left.\left(d N_{h} / d^{3} x\right)\right|_{\tau_{0}}=$ const. This assumption simplifies the problem significantly.

When $\sigma_{0}$ is a constant, the spatial dependence of the field potentials is immediately derived by using the Bessel-Fourier transform:

$$
\begin{aligned}
& \Phi\left(\tau, r_{T}\right)=\int_{0}^{\infty} \Phi_{0}\left(k_{T}\right) g_{\eta}\left(\tau, k_{T}\right) J_{0}\left(k_{T} r_{T}\right) d k_{T}, \\
& \Psi\left(\tau, r_{T}\right)=r_{T} \int_{0}^{\infty} \Psi_{0}\left(k_{T}\right) g_{\varphi}\left(\tau, k_{T}\right) J_{1}\left(k_{T} r_{T}\right) d k_{T},
\end{aligned}
$$

where the initial conditions resulting from CGC concept are applied:

$$
\begin{aligned}
& \left.g_{\eta}\right|_{\tau_{0}}=0,\left.\quad \frac{\partial_{\tau} g_{\eta}}{\tau}\right|_{\tau_{0}}=k_{T}, \\
& \left.g_{\varphi}\right|_{\tau_{0}}=1,\left.\quad \tau \partial_{\tau} g_{\varphi}\right|_{\tau_{0}}=0 .
\end{aligned}
$$

In principal, functions $g_{\eta}\left(\tau, k_{T}\right), g_{\varphi}\left(\tau, k_{T}\right)$ can be expressed for arbitrary $\tau_{0} \neq 0$ in terms of Heun functions. However, these expressions are complicated for heuristic analysis of our model and its applications. 
For this reason, we write down the functions $g_{\eta, \varphi}$ at $\tau_{0} \rightarrow 0:$

$$
\begin{aligned}
& g_{\eta}=-\frac{k_{T}}{2 \sigma_{0}} \exp \left(\frac{1}{2} \sigma_{0} \tau^{2}\right) M\left(-\frac{k_{T}^{2}}{4 \sigma_{0}}, \frac{1}{2} ;-\sigma_{0} \tau^{2}\right) \\
& g_{\varphi}=\frac{1}{\tau} \sqrt{\frac{2}{\sigma_{0}}} \exp \left(-\frac{1}{4} \sigma_{0} \tau^{2}\right) M\left(\frac{k_{T}^{2}-\sigma_{0}}{2 \sigma_{0}}, 0 ; \frac{1}{2} \sigma_{0} \tau^{2}\right)
\end{aligned}
$$

where $M(a, b ; z)$ is the Whittaker function.

It is easy to verify that the occurrence of negative color conductivity $\sigma_{\varphi}$ leads to a growth of the some components of magnetic and electric fields in comparison with the case of the theory without partonic medium. It is important that the Abelian magnetic field exhibits a growth, draining some energy from the particle reservoir. The instabilities are related with the presence of exponents in functions $g_{\eta, \varphi}$; Whittaker functions change actually a phase of oscillations only in comparison with the free theory.

If $\sigma_{0} \rightarrow 0$ and $\tau_{0} \rightarrow 0$, we come to the well-known expressions (see, for example, Ref. [27] and references therein):

$$
g_{\eta}\left(\tau, k_{T}\right)=\tau J_{1}\left(k_{T} \tau\right), \quad g_{\varphi}\left(\tau, k_{T}\right)=J_{0}\left(k_{T} \tau\right),
$$

where $J_{n}(z)$ is the Bessel function.

These expressions correspond to the perturbative (lowest order in the source charge densities) solution.

Now it is necessary to determine the functions $\Phi_{0}\left(k_{T}\right)$ and $\Psi_{0}\left(k_{T}\right)$. They originate from the initial conditions for the field equations.

Note that $\Psi_{0}\left(k_{T}\right)$ and $\Phi_{0}\left(k_{T}\right)$ are fluctuating quantities within the CGC concept, and the pair correlator of the (Yang-Mills) potentials is observable only. However, the field potentials in our approach are not stochastic quantities in the contrast with CGC ideology because we want to constitute the initial conditions on the base of the statistically averaged components of the energymomentum tensor accounting for the spatial inhomogeneity.

In order to derive $\Psi_{0}\left(k_{T}\right)$ and $\Phi_{0}\left(k_{T}\right)$, let us use the energy density distribution and the requirement of the absence of field flow at the initial moment. In mid-rapidity region $(\eta=0)$ and $\varphi=0$ (note that transverse directions are equal in the system with cylindrical symmetry), when $T_{t t}=T_{\tau \tau}, T_{t x}=T_{\tau x}$, we have that

$$
\begin{aligned}
\left.T_{t t}\right|_{\tau_{0}} & \equiv \varepsilon\left(r_{T}\right) \\
& =\frac{1}{2}\left(\left.\frac{\partial_{r_{T}} \Psi}{r_{T}}\right|_{\tau_{0}}\right)^{2}+\frac{1}{2}\left(\left.\frac{\partial_{\tau} \Phi}{\tau}\right|_{\tau_{0}}\right)^{2} \\
\left.T_{t x}\right|_{\tau_{0}} & =0
\end{aligned}
$$

where $\varepsilon\left(r_{T}\right)$ is assumed to be the known function from numerical calculations or physical point of view.

Our trick consists in the division of the energy density between different field components:

$$
\left.\partial_{r_{T}} \Psi\right|_{\tau_{0}}=\sqrt{\alpha} r_{T} f\left(r_{T}\right),\left.\quad \frac{\partial_{\tau} \Phi}{\tau}\right|_{\tau_{0}}=\sqrt{1-\alpha} f\left(r_{T}\right)
$$

where $f\left(r_{T}\right) \equiv \sqrt{2 \varepsilon\left(r_{T}\right)}$ and $\alpha$ is the separation constant (in general, $\alpha$ should be a function of $r_{T}$ ). Since the potentials $\Psi, \Phi$ are real, one has that $0 \leq \alpha \leq 1$.

In principal, $\alpha$ is an arbitrary constant. In fact it turns out that $\alpha \approx 1 / 2$ (it follows from a comparison of electric and magnetic strengths within the numerical approach). Note that the observables of the source-free theory are independent on $\alpha$.

Thus, one finds

$$
\Psi_{0}\left(k_{T}\right)=\sqrt{\alpha} \tilde{f}\left(k_{T}\right), \quad \Phi_{0}\left(k_{T}\right)=\sqrt{1-\alpha} \tilde{f}\left(k_{T}\right),
$$

here

$$
\tilde{f}\left(k_{T}\right)=\int_{0}^{\infty} f\left(r_{T}\right) J_{0}\left(k_{T} r_{T}\right) r_{T} d r_{T} .
$$

These expressions finally determine the fields in our model.

\section{APPLICATIONS}

In the previous Sections we have formulated the model of Glasma in a hard partonic medium. Since the classical field modes are usually interpreted as soft partons, their spatial dependence at an early stage of the $\mathrm{A}+\mathrm{A}$ collision may be done within the framework of the Glauber model. However, the explanations of experimental data can be efficiently with the application of other distributions too made. As was demonstrated in Ref. [24], the Gaussian distribution of soft partons leads to the adequate pion spectra produced after collision at RHIC. To formulate the field initial conditions, here we would like to choose the same approximation for the energy density at the initial moment,

$$
\varepsilon\left(r_{T}\right)=E \exp \left(-\frac{r_{T}^{2}}{2 R^{2}}\right)
$$

where $E=45 \mathrm{GeV} / \mathrm{fm}^{3}, R=3.768 \mathrm{fm}$.

Then we find that

$$
\tilde{f}\left(k_{T}\right)=2^{3 / 2} \sqrt{E} R^{2} \exp \left(-k_{T}^{2} R^{2}\right) .
$$

The boost-invariant distribution function $f^{0}$ (defining conductivities) is completely arbitrary at this point, so in order to proceed one needs to assume a specific form for it. In what follows we will require that $f^{0}$ is obtained from the isotropic function,

$$
N_{0} \exp \left(-\frac{p^{0}}{p_{h}}\right)
$$

by the replacement $y \rightarrow \theta$ in $p^{0}=p_{T} \cosh y$ and by the rescaling of one dimension in momentum space,

$$
f^{0}=N(\zeta) \exp \left(-\frac{p_{T}}{p_{h}} \sqrt{\cosh ^{2} \theta+\zeta \sinh ^{2} \theta}\right)
$$

where $p_{h}$ takes the role of saturation moment, $\zeta>-1$ is a parameter reflecting the strength of the partonic medium anisotropy and $N(\zeta)$ is a normalization constant. Note 
that $\zeta>0$ corresponds to a contraction of the distribution in the $z$-direction whereas $-1<\zeta<0$ corresponds to a stretching of the distribution in the $z$-direction.

Constant $N(\zeta)$ is simply determined by requiring the number density to be the same both for isotropic and anisotropic systems and can be evaluated (by integration over momentum variables) to be

$$
N(\xi)=N_{0} \sqrt{1+\zeta}
$$

Integrating over momentum, the multiplier defining the conductivities is

$$
\sigma_{0}=4 \pi g^{2} N_{0} p_{h}^{2} C(\zeta)
$$

where

$$
C(\zeta)=\frac{2}{3}(1+\zeta)^{3 / 2} F\left(\left[2, \frac{3}{2}\right],\left[\frac{5}{2}\right],-\zeta\right) .
$$

The coefficient $C(\zeta)$ in the region $\zeta \in(-1, \infty)$ is determined by a hypergeometric function $F$ and is such that $C(-1)=0$ (the case of source-free theory), $C(0)=2 / 3$ (for isotropic medium), $C(\infty)=\pi / 2$.

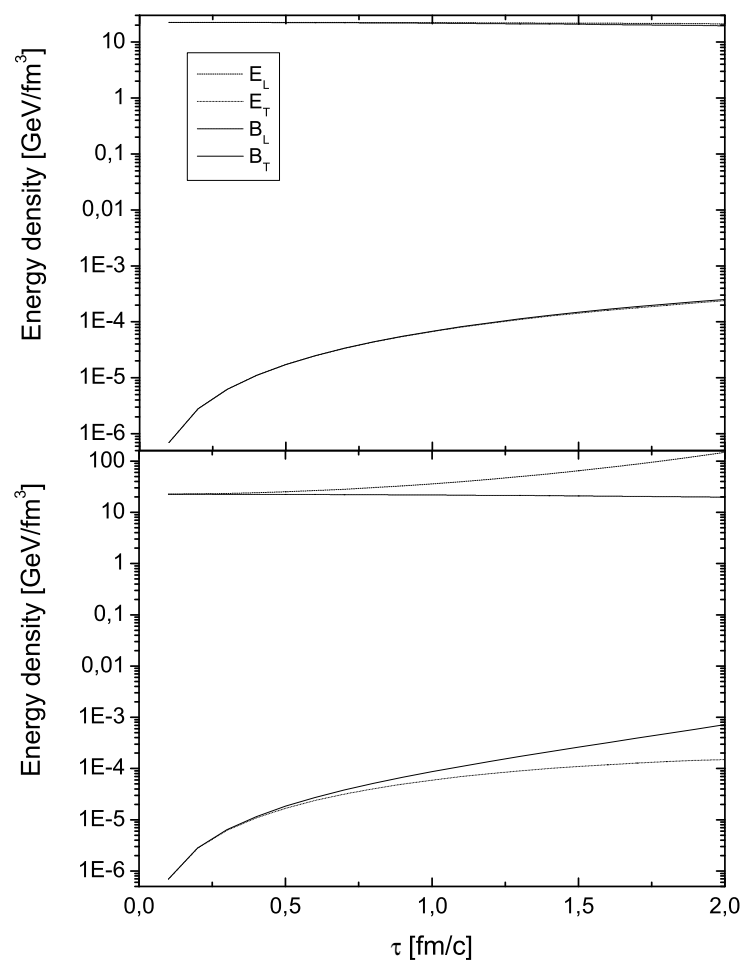

Fig. 1. Time evolution of the field energy density split into longitudinal and transverse electric $(E)$ and magnetic $(B)$ components at $r_{T}=0.1[\mathrm{fm}], \eta=0, \varphi=0$. The top panel corresponds to the free theory with $\sigma_{0}=0$. The bottom panel demonstrates the growth of $E_{L}$ and $B_{T}$ at $\sigma_{0}=0.25\left[\mathrm{fm}^{-2}\right]$.

Fig. 1 shows how the exponentially growing energy transferred from hard to soft partons is distributed among magnetic and electric fields at $\sigma_{0} \neq 0$ in comparison with the case of free field theory. The dominant contribution is still in longitudinal electric field (in accordance with CGC-like initial conditions). Nevertheless, we see that the transverse magnetic field demonstrates an unstable behavior too while this effect is absent in the free theory. Since the particle subsystem gives energy to the field, the total field energy density (as the sum of components) tends to grow with time.

Note that a similar model with expanding Abelian field coupled to the hard partons, when the strict boost invariance of fields is relaxed, has been already developed in Ref. [28] in the context of the quark-gluon plasma.

\section{CONCLUSIONS}

Generalizing the space-time evolution of the expanding Glasma with CGC-like initial conditions by the inclusion of small density of the hard partons anisotropically distributed in momentum space, we observe the instabilities (due to transferring energy from hard to soft partons) in the case of the abelianized boost-invariant model.

Here we propose to measure an anisotropy by means of transport coefficients like the conductivity tensor in the contrast with the usual approach based on the energymomentum tensor. As a result, the instabilities in the system under consideration lead to the conclusion of the presence of negative color conductivity in $\mathrm{A}+\mathrm{A}$ collisions. The sign of conductivity in transverse plane depends on the angle which speaks of a filamentation inherent to Weibel instabilities in plasma.

Unfortunately, the approximate solution derived to the transport equations does not permit us to achieve the isotropization here. This problem should be investigated in details and will be published elsewhere.

\section{ACKNOWLEDGEMENTS}

The research of the author was partially supported by the Foundation of Department of Physics and Astronomy of NAS of Ukraine.

\section{APPENDIX A. PROPAGATOR OF TRANSPORT EQUATION}

Here, we would like to discuss in detail the approximation which we have applied previously. To find $\delta f$, we have to determine operator $\hat{L}^{-1}$, inverse to the firstorder evolution operator $\hat{L}$. The inversion procedure of the evolution operator of transport equation was elaborated by Landau and, generally speaking, results in the emergence of the Landau damping in plasma.

Let $G$ is the solution of the following equation:

$$
\hat{L} G\left(\tau, \theta, \mathbf{r}_{T} \mid \tau^{\prime}, \theta^{\prime}, \mathbf{r}_{T}^{\prime}\right)=\frac{\delta\left(\tau-\tau^{\prime}\right)}{\tau} \delta\left(\theta-\theta^{\prime}\right) \delta^{2}\left(\mathbf{r}_{T}-\mathbf{r}_{T}^{\prime}\right),
$$

where the right hand side is the 4-dimensional $\delta$-function with respect to the pseudo-cylindric measure $\tau d \tau d \theta d^{2} r_{T}$.

Without loosing generality, we limit ourselves by the case, when $\tau_{0} \rightarrow 0$, and by using the transverse coordinates $\mathbf{r}_{T}$ instead of cylindrical $\left(r_{T}, \varphi\right)$.

Using the formulas from Appendix B, $G$ is represented as 


$$
G\left(\tau, \theta, \mathbf{r}_{T} \mid \tau^{\prime}, \theta^{\prime}, \mathbf{r}_{T}^{\prime}\right)=\lim _{\varepsilon \rightarrow+0} \frac{i}{(2 \pi)^{4} p_{T}} \int \frac{\mathrm{e}^{i \omega\left[\tau \cosh (\xi-\theta)-\tau^{\prime} \cosh \left(\xi-\theta^{\prime}\right)\right]-i \mathbf{k}_{T}\left(\mathbf{r}_{T}-\mathbf{r}_{T}^{\prime}\right)}}{\mathbf{k}_{T} \mathbf{v}_{T}-\omega \cosh \xi-i \varepsilon} d \omega d \xi d^{2} k_{T}
$$

where $\mathbf{v}_{T} \equiv \mathbf{p}_{T} / p_{T}$ and the Landau damping is already taken into account due to auxiliary formula:

$$
\lim _{\varepsilon \rightarrow+0} \frac{1}{x-i \varepsilon}=\mathcal{P} \frac{1}{x}+i \pi \delta(x)
$$

Discarding the spatial dispersion, we have to assume that $k_{T} \ll \omega \cosh \xi$. It leads to simplification:

$$
\begin{aligned}
& G\left(\tau, \theta, \mathbf{r}_{T} \mid \tau^{\prime}, \theta^{\prime}, \mathbf{r}_{T}^{\prime}\right) \approx \frac{1}{p_{T}} \Theta\left(\tau \cosh \theta-\tau^{\prime} \cosh \theta^{\prime}\right) \\
& \times \delta\left(\tau \sinh \theta-\tau^{\prime} \sinh \theta^{\prime}\right) \delta^{2}\left(\mathbf{r}_{T}-\mathbf{r}_{T}^{\prime}\right)
\end{aligned}
$$

where $\Theta$ is Heaviside function defined as $\Theta(x<0)=0$, $\Theta(x=0)=1 / 2, \Theta(x>0)=1$.

This approximation says that the system is homogeneous at significantly large times and the fluctuations are localized in space.

Furthermore, let the Björken scaling flow, when $\theta \approx 0$, take place. It means that $\theta$ and $\theta^{\prime}$ should be equal and gives us that

$$
G\left(\tau, \theta, \mathbf{r}_{T} \mid \tau^{\prime}, \theta^{\prime}, \mathbf{r}_{T}^{\prime}\right) \approx \frac{\Theta\left(\tau-\tau^{\prime}\right)}{p_{T} \tau^{\prime} \cosh \theta^{\prime}} \delta\left(\theta-\theta^{\prime}\right) \delta^{2}\left(\mathbf{r}_{T}-\mathbf{r}_{T}^{\prime}\right) .
$$

More precisely, it can be derived from condition, $\tau \sinh \theta=$ const, resulting in

$$
\sinh \theta d \tau+\tau \cosh \theta d \theta=0
$$

where $d \tau=\tau-\tau^{\prime}, d \theta=\theta-\theta^{\prime}$.

Assuming that $\tau$ is small and the expression under integration is not essentially changed at this time range, we can do the following replacement:

$$
\int_{0}^{\infty} d \tau^{\prime} \Theta\left(\tau-\tau^{\prime}\right) F\left(\tau^{\prime}\right) \rightarrow \tau F(\tau)
$$

Then, one obtains that

$$
\begin{aligned}
& \int G\left(\tau, \theta, \mathbf{r}_{T} \mid \tau^{\prime}, \theta^{\prime}, \mathbf{r}_{T}^{\prime}\right) F\left(\tau^{\prime}, \theta^{\prime}, \mathbf{r}_{T}^{\prime}\right) \tau^{\prime} d \tau^{\prime} d \theta^{\prime} d^{2} r_{T}^{\prime} \approx \\
& \approx \frac{\tau}{p_{T} \cosh \theta} F\left(\tau, \theta, \mathbf{r}_{T}\right) .
\end{aligned}
$$

This formula determines the solution of inhomogeneous transport equation with the source $F$.

\section{APPENDIX B. INTEGRAL TRANSFORMATION}

The Fourier transformation reads

$$
f(t, z)=\int_{-\infty}^{\infty} \frac{d \mu d \mu^{\prime}}{(2 \pi)^{2}} \int_{-\infty}^{\infty} f(p, s) \mathrm{e}^{i \mu(t-p)-i \mu^{\prime}(z-s)} d p d s .
$$

Let us introduce new variables:

$$
\begin{aligned}
& t=\tau \cosh \theta, \quad z=\tau \sinh \theta \\
& p=\rho \cosh \psi, \quad s=\rho \sinh \psi \\
& \mu=\lambda \cosh \phi, \quad \mu^{\prime}=\lambda \sinh \phi
\end{aligned}
$$

If $f(t, z)=F(\tau, \theta)$, one gets the following transformation rule:

$$
\begin{gathered}
\tilde{F}(\lambda, \phi)=\int_{-\infty}^{\infty} F(\rho, \psi) \mathrm{e}^{-i \rho \lambda \cosh (\phi-\psi)} \rho d \rho d \psi, \\
F(\tau, \theta)=\frac{1}{(2 \pi)^{2}} \int_{-\infty}^{\infty} \tilde{F}(\lambda, \phi) \mathrm{e}^{i \tau \lambda \cosh (\phi-\theta)} \lambda d \lambda d \phi .
\end{gathered}
$$

For example, we find that

$\frac{1}{\tau} \delta(\Delta \tau) \delta(\Delta \theta)=\frac{1}{(2 \pi)^{2}} \int_{-\infty}^{\infty} \mathrm{e}^{i \lambda\left[\tau \cosh (\phi-\Delta \theta)-\tau_{0} \cosh \phi\right]} \lambda d \lambda d \phi$

where $\Delta \tau=\tau-\tau_{0}, \Delta \theta=\theta-\theta_{0}$.
[1] I. Arsene et al. [BRAHMS collaboration], Nucl. Phys. A 757, 1 (2005); B. B. Back et al. [PHOBOS collaboration], ibid, 28 (2005); J. Adams et al. [STAR collaboration], ibid, 102 (2005); K. Adcox et al. [PHENIX collaboration], ibid, 184 (2005).

[2] S. Mrówczyński, Phys. Lett. B 314, 118 (1993); Phys. Rev. C 49, 2191 (1994); Phys. Lett. B 393, 26 (1997).

[3] S. Mrówczyński, Acta Phys. Pol. B 37427 (2006).

[4] V. M. Emelyanov, S. L. Timoshenko, M. N. Strihanov Introduction in relativistic nuclear physics (Fizmatlit,
Moscow, 2004) (in Russian).

[5] F. Gelis, R. Venugopalan, Acta Phys. Pol. B 37, 3253 (2006); arXiv: hep-ph/0611157 (2006).

[6] L. McLerran, R. Venugopalan, Phys. Rev. D 49, 2233, 3352 (1994); 50, 2225 (1994); 53, 458 (1996); 59, 09400 (1999).

[7] A.V. Leonidov, Phys. Usp. 48, 323 (2005).

[8] E.S. Weibel, Phys. Rev. Lett. 2, 83 (1959).

[9] P. Romatschke, R. Venugopalan, Phys. Rev. Lett. 96, 062302 (2006). 
[10] K. Geiger, B. Mueller, Nucl. Phys. B 369600 (1992).

[11] S. A. Bass, B. Mueller, D. K. Srivastava, Phys. Lett. B 551, 277 (2003).

[12] M. Gyulassy, Y. Pang, B. Zhang, Nucl. Phys. A 626, 999 (1997).

[13] D. Molnar, M. Gyulassy, Phys. Rev. C 62, 054907 (2000).

[14] A. Dumitru, M. Gyulassy, Phys. Lett. B 494, 215 (2000).

[15] Y. Nara, S. E. Vance, P. Csizmadia, Phys. Lett. B 531, 209 (2002).

[16] Z. Xu, C. Greiner, Phys. Rev. C 71, 064901 (2005).

[17] R. Baier, A. H. Mueller, D. Schiff, D. T. Son, Phys. Lett. B 502, 51 (2001).

[18] S. V. Akkelin, Phys. Rev. C 78, 014906 (2008).

[19] A. Dumitru, Y. Nara, Phys. Lett. B 621, 89 (2005).

[20] S. Mrówczyński, A. Rebhan, M. Strickland, Phys. Rev. D 70, 025004 (2004).
[21] P. Arnold, J. Lenaghan, Phys. Rev. D 70, 114007 (2004).

[22] M. Gyulassy, Iu. Karpenko, A. V. Nazarenko, Yu. M. Sinyukov, Braz. J. Phys. 37, 1031 (2007).

[23] Yu. M. Sinyukov, A. V. Nazarenko, Iu. A. Karpenko, Acta Phys. Pol. B 40, 1109 (2009).

[24] Yu. M. Sinyukov, Iu. A. Karpenko, A. V. Nazarenko, J. Phys. G: Nucl. Part. Phys. 35, 104071 (2008).

[25] R. J. Fries, J. I. Kapusta, Y. Li, Nucl. Phys. A 774, 861 (2006).

[26] P. Arnold, J. Lenaghan, G. D. Moore, L. G. Yaffe, Phys. Rev. Lett. 94, 072302 (2005).

[27] T. Lappi, Phys. Lett. B 643, 11 (2006); arXiv: hep$\mathrm{ph} / 0606207$ (2006).

[28] P. Romatschke, A. Rebhan, Phys. Rev. Lett. 97, 252301 (2006); hep-ph/0605064 (2006).

\title{
ЕВОЛЮЦІЯ ГЛАЗМИ У ПАРТОННОМУ СЕРЕДОВИЩІ
}

\author{
А. В. Назаренко \\ Інститут теоретичної фізики ім. М. М. Боголюбова, \\ вул. Метрологічна, 14-б, Київ, 03680, Україна
}

\begin{abstract}
Ми досліджуємо сценарій еволюції глазми з урахуванням зворотної дії партонного середовища в ультрарелятивістичних зіткненнях важких йонів. Ми анонсуємо, що таке узагальнення приводить до нестійкостей та наявності від'ємної кольорової провідності у системі.
\end{abstract}

